\title{
A Pitfall in the Diagnosis of Eosinophilic Myocarditis in a Patient who Received Steroid Therapy
}

\author{
Yusuke Watanabe, Hiroshi Wada, Kenichi Sakakura, Hideo Fujita and Shin-ichi Momomura
}

\begin{abstract}
Eosinophilic myocarditis is a rare form of myocardial inflammation that is characterized by the infiltration of eosinophilic cells into the myocardium. The clinical symptoms of eosinophilic myocarditis are similar to those of acute coronary syndrome, and eosinophilic myocarditis sometimes occurs in combination with bronchial asthma. We herein present a case of eosinophilic myocarditis in which additional time was required to make a definitive diagnosis because the patient received steroid therapy. The diagnosis of eosinophilic myocarditis is challenging, especially when a patient has other inflammatory diseases, such as bronchial asthma. We should pay attention to the possibility that steroid therapy may mask the presentation of eosinophilic myocarditis.
\end{abstract}

Key words: eosinophilic myocarditis, bronchial asthma, chest pain, steroid

(Intern Med 56: 157-161, 2017)

(DOI: 10.2169/internalmedicine.56.7521)

\section{Introduction}

Eosinophilic myocarditis is a rare form of myocardial inflammation that is characterized by the infiltration of eosinophilic cells into the myocardium (1). The clinical symptoms of eosinophilic myocarditis are similar to those of acute coronary syndrome, and eosinophilic myocarditis sometimes occurs in combination with bronchial asthma $(2,3)$. The anti-inflammatory effects of the steroids that are prescribed to bronchial asthma patients may interfere with the correct diagnosis of other inflammatory diseases. We herein present a case in which additional time was required to make a definitive diagnosis of eosinophilic myocarditis, because the patient had been treated with steroids.

\section{Case Report}

A 77-year-old woman with a history of asthma was admitted to the coronary care unit (CCU) of our hospital due to the sudden onset of severe chest pain. An electrocardiogram showed slight ST depression in the V4, V5 and V6 leads (Fig. 1a). An echocardiogram showed mild hypokinesis of the anterior wall and slight pericardial effusion. Con- trast enhanced computed tomography (CT), which was performed after bolus steroid infusion (methylprednisolone, mPSL) to prevent an asthma attack, showed no abnormal findings (such as acute aortic dissection or acute pulmonary embolism). A blood analysis that was performed in the emergency room showed eosinophilia $(8,555 \mathrm{cells} / \mu \mathrm{L}, 58 \%$ of the white blood cell count) prior to the steroid infusion. However, the patient's eosinophilia improved remarkably to 1,363 cells $/ \mu \mathrm{L}$ ( $15 \%$ of the white blood cell count) following the bolus infusion of methylprednisolone $(125 \mathrm{mg})$ at approximately 4 hours after admission. Although eosinophilic myocarditis should have been suspected as one of the differential diagnoses, we did not consider eosinophilic myocarditis to be a likely diagnosis because the patient's eosinophilia showed a marked improvement following the infusion of methylprednisolone, which was administered as prophylaxis against bronchial asthma. Coronary angiography (CAG) showed no significant organic stenosis (Fig. 1b). Left ventriculography (LVG) showed mild hypokinesis of the anterior midwall (Fig. 1c). Based on the results of CAG and LVG, acute coronary syndrome associated with coronary spastic angina of the left anterior descending (LAD) artery territory was suspected. We did not perform an acetylcholine provocation test because acetylcholine is contraindicated in 


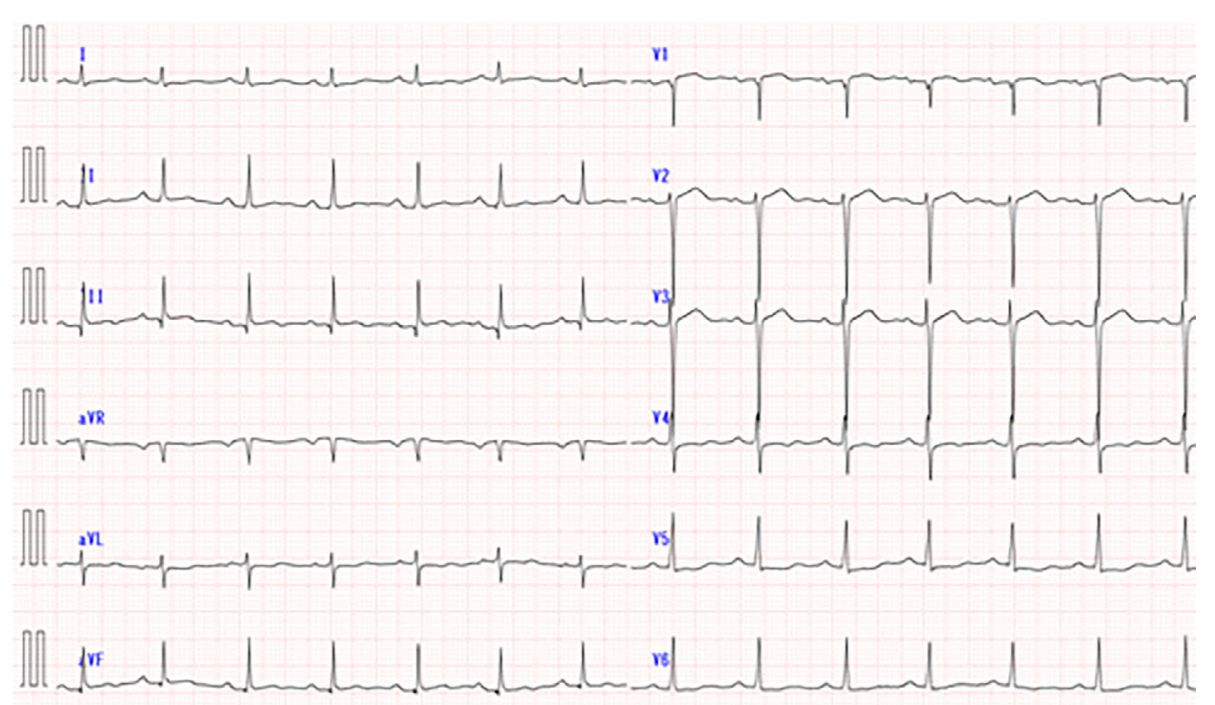

Figure 1a. The electrocardiogram on first admission showed slight ST depression in the V4, V5 and V6 leads.
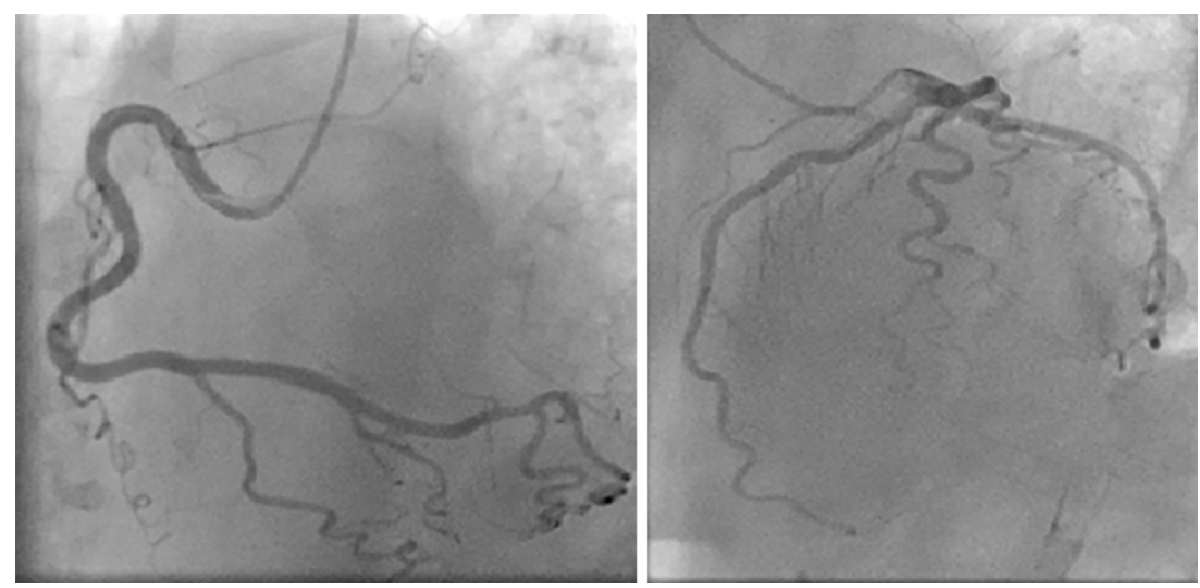

Figure 1b. Coronary angiography revealed a normal coronary artery.

patients with bronchial asthma. Based on the regional hypokinesis of the left ventricle, the differential diagnoses included sarcoidosis, amyloidosis, and takotsubo-cardiomyopathy. We excluded sarcoidosis and amyloidosis, because her chest pain was not typical for either condition. Furthermore, an echocardiogram did not show left ventricular hypertrophy (suggestive of amyloidosis) or basal intraventricular wall thinning (suggestive of sarcoidosis). We also excluded takotsubo-cardiomyopathy because the findings of left ventriculography were atypical for takotsubocardiomyopathy and because the patient's electrocardiogram showed no typical changes such as giant negative $\mathrm{T}$ waves in the precordial leads. Her symptoms improved within a few days after the administration of a calcium channel blocker. We therefore diagnosed the patient with coronary spastic angina complicated with bronchial asthma at the time of the first hospitalization. She was discharged on foot, and was followed up in our outpatient clinic.

A month later her chest pain recurred, and she was readmitted to CCU. A blood analysis revealed the marked eleva- tion of troponin I (11,906 pg/mL) and eosinophilia $(15,262$ cells $/ \mu \mathrm{L}, 62.5 \%$ ). An echocardiogram showed mild hypokinesis of the anterior wall and a slight increase in pericardial effusion (Fig. 1d). Based on her clinical course, we suspected myocarditis, and performed an endomyocardial biopsy, which showed remarkable eosinophilic infiltration in the interstitium of the myocardium (Fig. 2). An extensive work-up for the patient's secondary eosinophilia did not show any specific findings. We therefore diagnosed the patient with eosinophilic myocarditis, and prescribed prednisolone $(1 \mathrm{mg} / \mathrm{kg} / \mathrm{day})$. Following the initiation of prednisolone treatment, the patient's eosinophilic cell count and her troponin I level dropped remarkably ( 7 cell $/ \mu \mathrm{L}, 26 \mathrm{pg} / \mathrm{mL}$ ). After this treatment, her clinical symptoms completely disappeared (Fig. 3).

\section{Discussion}

The present case demonstrates the difficulty in diagnosing eosinophilic myocarditis in patients with bronchial asthma. 

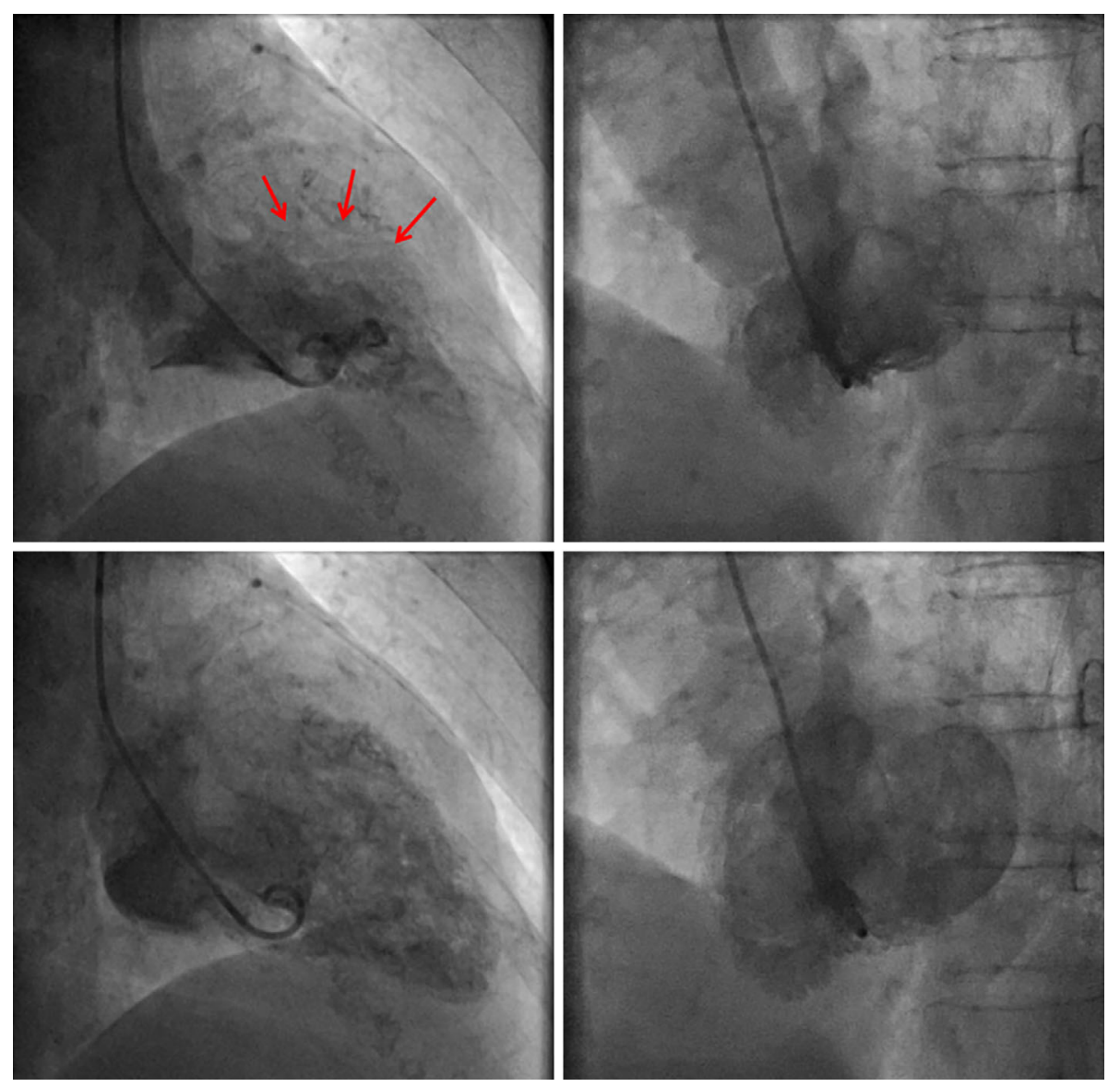

Figure 1c. Left ventriculography showed mild hypokinesis of the anterior wall (arrows). The two images on the left side show right anterior oblique (RAO) 30-degree views. The two images on the right side show left anterior oblique (LAO) 60-degree views.
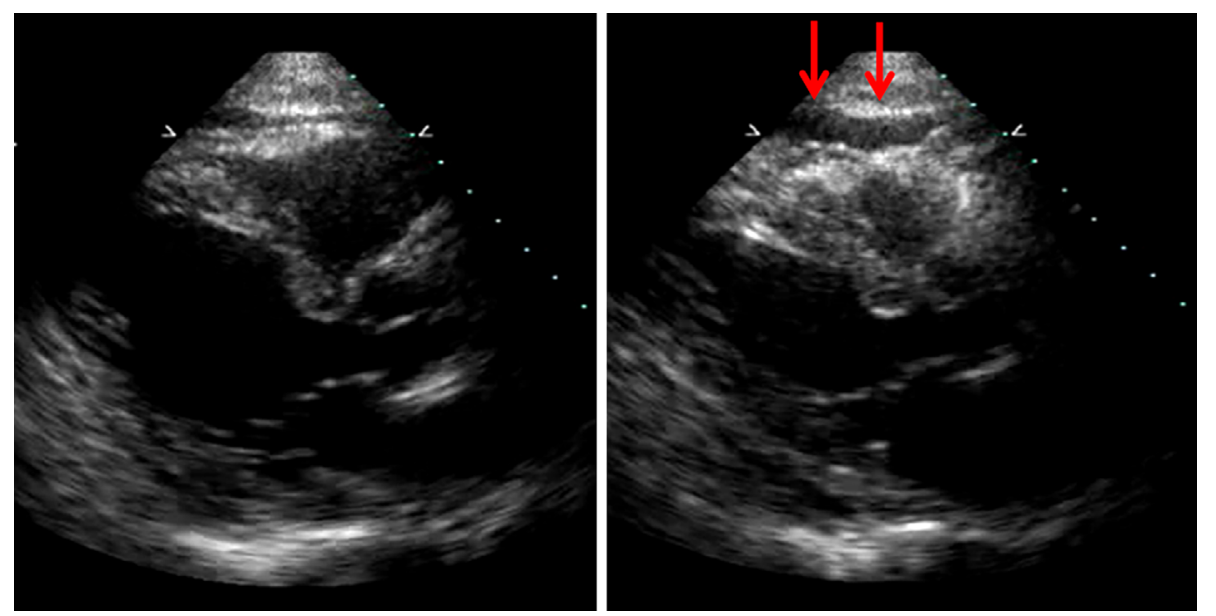

Figure 1d. An echocardiogram showed mild hypokinesis of the anterior wall and a slight increase in pericardial effusion. The arrows indicate pericardial effusion.

First, since the peripheral blood sample of a patient with bronchial asthma often shows eosinophilia (4), we did not consider eosinophilia as a sign of eosinophilic myocarditis. It is important to suspect eosinophilic myocarditis in patients with chest pain and eosinophilia, even if they have other diseases that are associated with eosinophilia, such as bronchial asthma, collagen disease, or hematological disor- ders (5). Second, since steroid therapy often normalizes peripheral eosinophilia $(6,7)$, it was difficult to consider eosinophilic myocarditis as one of the differential diagnoses in this patient, due to the normalization of her peripheral blood cell count. It is important to suspect eosinophilic myocarditis in patients with chest pain and without coronary artery disease, if they receive steroid therapy that normalizes 
peripheral eosinophilia.

The previous literature reports that one third of patients with eosinophilic myocarditis have a history of allergy or asthma (8). Furthermore, the European Society of Urogenital Radiology (ESUR) guidelines recommend the use of steroids for preventing asthma attacks when contrast media is administered to asthma patients (9). Thus, the present case does not represent a rare situation.

Eosinophilic myocarditis is a rare form of myocarditis

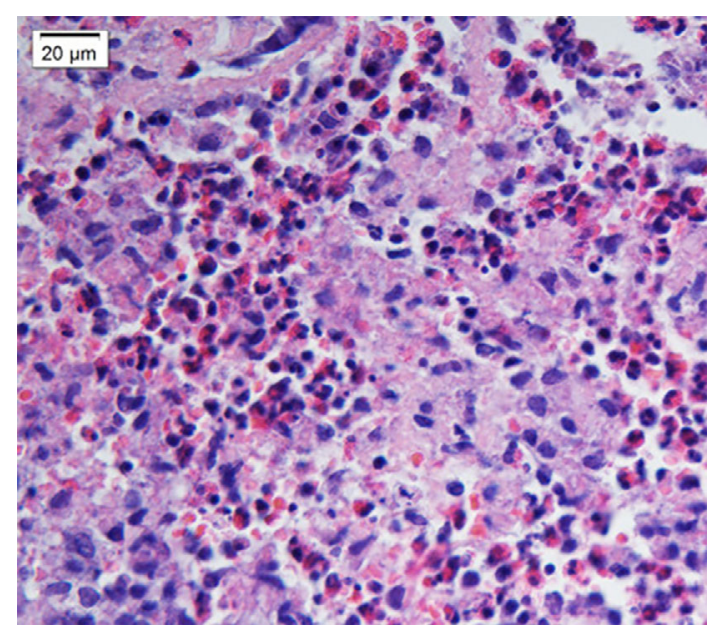

Figure 2. An endomyocardial biopsy specimen showed remarkable eosinophilic cell infiltration at the interstitium of the myocardium and necrosis of the myocardium. This findings were compatible with eosinophilic myocarditis (Hematoxylin and Eosin staining, $\times 200$ ). characterized by diffuse or focal myocardial inflammation with eosinophilic infiltration (10). While eosinophilia is often observed in peripheral blood samples, a definite diagnosis requires evidence of eosinophilic infiltration in the myocardium (11). However, since eosinophilic infiltration sometimes only affects the focal myocardium, the sensitivity of endomyocardial biopsy is insufficient (approximately $50 \%)(2,10)$. Positron emission tomography (PET) and gallium scintigraphy have been reported to be useful as noninvasive modalities for diagnosing eosinophilic myocarditis $(5,10)$. Thus, these non-invasive modalities can help in the diagnosis of eosinophilic myocarditis, especially when the results of an endomyocardial biopsy are inconclusive.

This case showed several clinical features that were suggestive of acute coronary syndrome. There are some reports of cases in which eosinophilic myocarditis showed a similar presentation to acute myocardial infarction $(2,11)$. There are often abnormalities (usually ST-T segment abnormalities) in the electrocardiograms of patients with eosinophilic myocarditis (5). Thus, a diagnostic procedure that includes myocardial biopsy should be positively considered when no significant coronary artery stenosis is found in patients with the clinical presentation of acute coronary syndrome.

In conclusion, the diagnosis of eosinophilic myocarditis is challenging, especially when a patient has other inflammatory disease such as bronchial asthma. We should pay attention to the possibility that steroid therapy may mask the presentation of eosinophilic myocarditis.

Written informed consent was obtained from patient for the publication of this case report and any accompanying

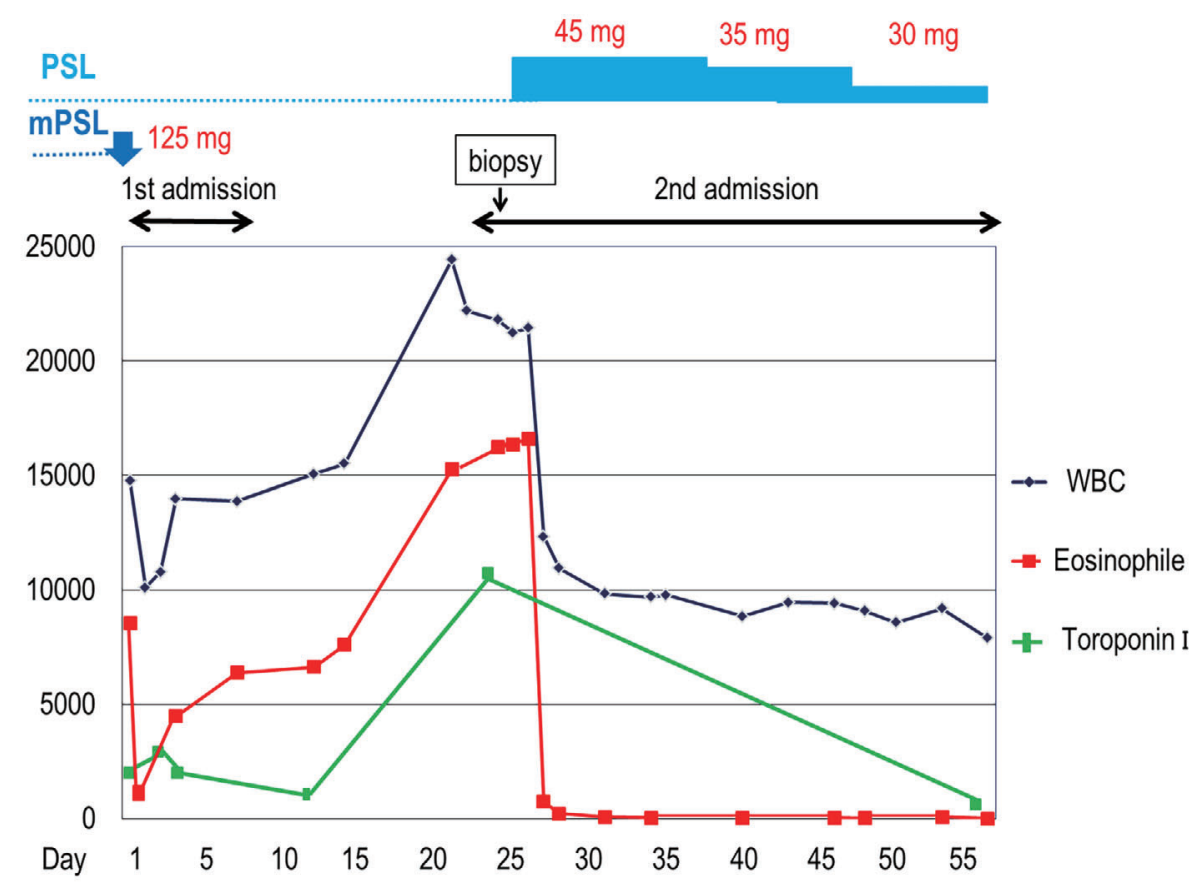

Figure 3. The clinical course before and after prednisolone treatment. The infusion of methylprednisolone $(125 \mathrm{mg})$ temporarily improved the patient's symptoms, eosinophilia and troponin I level on her first admission. The patient's eosinophilia and troponin I level showed a complete improvement after the administration of PSL during the patient's 2nd admission. 
images.

The authors state that they have no Conflict of Interest (COI).

\section{References}

1. Ammirati E, Stucchi M, Brambatti M, et al. Eosinophilic myocarditis: a paraneoplastic event. Lancet 385: 2546, 2015.

2. Amini R, Nielsen C. Eosinophilic myocarditis mimicking acute coronary syndrome secondary to idiopathic hypereosinophilic syndrome: a case report. J Med Case Rep 4: 40, 2010.

3. Galiuto L, Enriquez-Sarano M, Reeder GS, et al. Eosinophilic myocarditis manifesting as myocardial infarction: early diagnosis and successful treatment. Mayo Clin Proc 72: 603-610, 1997.

4. Eng SS, DeFelice ML. The role and immunobiology of eosinophils in the respiratory system: a comprehensive review. Clin Rev Allergy Immunol 50: 140-158, 2016.

5. Kuchynka P, Palecek T, Masek M, et al. Current diagnostic and therapeutic aspects of eosinophilic myocarditis. BioMed Res Int 2016: 2829583, 2016.

6. Yanagisawa $T$, Inomata $T$, Watanabe $I$, et al. Clinical significance of corticosteroid therapy for eosinophilic myocarditis. Int Heart $\mathrm{J}$
52: 110-113, 2011.

7. Eppenberger M, Hack D, Ammann P, Rickli H, Maeder MT. Acute eosinophilic myocarditis with dramatic response to steroid therapy: the central role of echocardiography in diagnosis and follow-up. Tex Heart Instit J 40: 326-330, 2013.

8. Kawano S, Kato J, Kawano N, et al. Clinical features and outcomes of eosinophilic myocarditis patients treated with prednisolone at a single institution over a 27-year period. Intern Med 50: 975-981, 2011.

9. Morcos SK, Thomsen HS, Webb JA. Prevention of generalized reactions to contrast media: a consensus report and guidelines. Eur Radiol 11: 1720-1728, 2001.

10. Rizkallah J, Desautels A, Malik A, et al. Eosinophilic myocarditis: two case reports and review of the literature. BMC Res Notes 6 : 538, 2013.

11. Enriquez A, Castro P, Gabrielli L, et al. Acute necrotizing eosinophilic myocarditis presenting as ST-elevation myocardial infarction: a case report. Can J Cardiol 27: 870.e1-870.e3, 2011.

The Internal Medicine is an Open Access article distributed under the Creative Commons Attribution-NonCommercial-NoDerivatives 4.0 International License. To view the details of this license, please visit (https://creativecommons.org/licenses/ by-nc-nd/4.0/).

(C) 2017 The Japanese Society of Internal Medicine http://www.naika.or.jp/imonline/index.html 